\title{
A randomized, 3-arm, neoadjuvant, phase 2 study comparing docetaxel + carboplatin + trastuzumab + pertuzumab (TCbHP), TCbHP followed by trastuzumab emtansine and pertuzumab (T-DM1+P), and T-DM1+P in HER2-positive primary breast cancer
}

\author{
Norikazu Masuda ${ }^{1}$ (D) Shoichiro Ohtani ${ }^{2} \cdot$ Toshimi Takano $^{3} \cdot$ Kenichi Inoue $^{4} \cdot$ Eiji Suzuki $^{5} \cdot$ Rikiya Nakamura $^{6}$. \\ Hiroko Bando ${ }^{7}$ Yoshinori Ito ${ }^{8} \cdot$ Kazushige Ishida $^{9} \cdot$ Takashi Yamanaka $^{10} \cdot$ Katsumasa Kuroi $^{11} \cdot$ Hiroyuki Yasojima $^{1}$. \\ Hiroi Kasai ${ }^{12} \cdot$ Tsuyoshi Takasuka $^{13} \cdot$ Takaki Sakurai $^{14} \cdot$ Tatsuki R. Kataoka $^{14} \cdot$ Satoshi Morita $^{15} \cdot$ Shinji Ohno $^{16}$. \\ Masakazu Toi ${ }^{17}$ (D)
}

Received: 1 November 2019 / Accepted: 8 January 2020 / Published online: 17 January 2020

(c) The Author(s) 2020

\begin{abstract}
Purpose The standard of care in the neoadjuvant setting for human epidermal growth factor receptor 2 (HER2)-positive breast cancer is dual HER2-targeted therapy. However, a need to minimize treatment-related toxicity and improve pathological complete response (pCR) rates, particularly in luminal HER2-positive disease, exists.

Methods Neopeaks, a randomized, phase 2 study, compared docetaxel + carboplatin + trastuzumab + pertuzumab (TCbHP; 6 cycles; group A), TCbHP (4 cycles) followed by trastuzumab emtansine + pertuzumab (T-DM1+P; 4 cycles; group B), and T-DM1+P (4 cycles; group $\mathrm{C}$ ) regimens in HER2-positive primary breast cancer patients; concurrent hormone therapy with $\mathrm{T}-\mathrm{DM} 1+\mathrm{P}$ was administered in case of estrogen receptor positivity $(\mathrm{ER}+)$. Based on tumor shrinkage, nonresponders in group $\mathrm{C}$ were switched to 5-fluorouracil + epirubicin + cyclophosphamide (FEC; 4 cycles). Primary endpoint was pCR (comprehensive pCR ypN0 [ypT0-TisypN0]).

Results Of 236 patients enrolled, 204 were randomized to groups A $(n=51), \mathrm{B}(n=52)$, and C $(n=101)$. In group C, 80 (79\%) patients continued T-DM1+P following favorable response, whereas $21(21 \%)$ nonresponders switched to FEC. pCR rate was numerically higher with the TCbHP $\rightarrow$ T-DM1+P regimen $(71 \%)$ versus the standard TCbHP $(57 \%)$ and T-DM1+P $(57 \%)$ regimens. The rate in group $\mathrm{C}$ was higher among responders continuing T-DM1+P (63\%) versus nonresponders who switched to FEC (38\%). pCR rates after initial 4 cycles of T-DM1+P (group C; 57\%) and standard TCbHP regimen (57\%) were equivalent. pCR rate in patients with ER+ was significantly higher in group B (69\%) than groups A (43\%) and C (51\%), but was comparable in patients with ER- (67-76\%). Compared with the T-DM1-based arm, the incidence of adverse events was higher in the taxane-based arms.

Conclusion In the neoadjuvant setting, the pCR rate with the standard TCbHP $\rightarrow$ T-DM1+P regimen was numerically better than the TCbHP regimen alone and significantly better in patients with ER+. Personalization of the T-DM1+P regimen could serve as a reasonable approach to minimize toxicity while maintaining efficacy.
\end{abstract}

Trial registration ID: UMIN-CTR: UMIN000014649.

Keywords Dual HER2-targeted therapy · Neoadjuvant therapy $\cdot$ Pertuzumab $\cdot$ Trastuzumab emtansine $\cdot$ Pathological complete response $\cdot$ Safety

Electronic supplementary material The online version of this article (https://doi.org/10.1007/s10549-020-05524-6) contains supplementary material, which is available to authorized users.

Masakazu Toi

toi@kuhp.kyoto-u.ac.jp

Extended author information available on the last page of the article

$\begin{array}{ll}\text { Abbreviations } \\ \mathrm{AE} & \text { Adverse event } \\ \mathrm{AI} & \text { Aromatase inhibitor } \\ \mathrm{BCS} & \text { Breast-conserving surgery } \\ \mathrm{Bp} & \text { Partial mastectomy } \\ \mathrm{Bq} & \text { Quadrantectomy } \\ \mathrm{cCR} & \text { Clinical complete response }\end{array}$




$\begin{array}{ll}\text { CI } & \text { Confidence interval } \\ \text { CISH } & \text { Chromogenic in situ hybridization } \\ \text { CpCR } & \text { Comprehensive pCR } \\ \text { DISH } & \text { Dual in situ hybridization } \\ \text { DFS } & \text { Disease-free survival } \\ \text { ER } & \text { Estrogen receptor } \\ \text { FEC } & \text { 5-Fluorouracil + epirubicin + cyclophos- } \\ & \text { phamide } \\ \text { FISH } & \text { Fluorescence in situ hybridization } \\ \text { HER2 } & \text { Human epidermal growth factor receptor 2 } \\ \text { HR } & \text { Hormone receptor } \\ \text { HT } & \text { Hormone therapy } \\ \text { IHC } & \text { Immunohistochemistry } \\ \text { JBCRG } & \text { Japan Breast Cancer Research Group } \\ \text { LHRH } & \text { Luteinizing hormone-releasing hormone } \\ \text { MedDRA/J } & \text { Medical dictionary for regulatory } \\ & \text { activities/japanese } \\ \text { MRI } & \text { Magnetic resonance imaging } \\ \text { NCI CTCAE } & \text { National cancer institute common termi- } \\ & \text { nology criteria for adverse events } \\ \text { ORR } & \text { Overall response rate } \\ \text { OS } & \text { Overall survival } \\ \text { pCR } & \text { Pathological complete response } \\ \text { pCRinv } & \text { Invasive pCR } \\ \text { PET-CT } & \text { Positron emission tomography-computed } \\ & \text { tomography } \\ \text { PFS } & \text { Progression-free survival } \\ \text { PgR } & \text { Progesterone receptor } \\ \text { QpCR } & \text { Quasi pCR } \\ \text { RDI } & \text { Relative dose intensity } \\ \text { RECIST } & \text { Response evaluation criteria in solid } \\ & \text { tumors } \\ \text { SpCR } & \text { Strict pCR } \\ \text { TCbHP } & \text { Docetaxel + carboplatin + trastu- } \\ & \text { zumab + pertuzumab } \\ \text { T-DM1 } & \text { Trastuzumab emtansine } \\ \text { T-DM1+P } & \text { T-DM1+Pertuzumab } \\ & \end{array}$

\section{Introduction}

Current treatment guidelines recommend the use of multidrug chemotherapy, such as a sequential combination of an anthracycline-containing regimen and taxane or concurrent use of taxane and platinum, in combination with the antihuman epidermal growth factor receptor 2 (HER2) monoclonal antibodies trastuzumab and pertuzumab for the treatment of HER2-positive (HER2+) primary breast cancer [1, 2].

Dual HER2-targeted therapy with lapatinib or pertuzumab in combination with trastuzumab has significantly increased pathological response rates in patients with HER2+ breast cancer. For example, ypT0/is and/or ypN0 rates (pathological complete response $[\mathrm{pCR}]$ ) showed improvement from
$29.5-52.5 \%$ to $51.3-65.9 \%$ by adding lapatinib [3-6] and from $22-29 \%$ to $45.8-66.2 \%$ by adding pertuzumab [7-10]. Furthermore, attempts have been made to reduce the toxicity of chemotherapy by using dual HER 2 blockade alone or trastuzumab emtansine (T-DM1) with or without pertuzumab. Dual HER2 blockade with trastuzumab and pertuzumab alone in the absence of cytotoxic chemotherapy has shown reasonable pathological response rates $(16.8 \%)$ in patients with locally advanced or inflammatory, operable, HER2+ breast cancer [7]. The response rate was higher (44.4\%) with the combination of T-DM1 and pertuzumab (T-DM1+P) in patients with HER2+ early breast cancer [10].

Several studies have investigated HER2 blockade with concurrent hormone therapy (HT) in patients with hormone receptor-positive (HR+) HER2+ breast cancer. A phase 3 study demonstrated reduced disease progression (hazard ratio $0.71 ; 95 \%$ confidence interval [CI] 0.53-0.96; $P=0.019$ ) and significant clinical benefit with the lapatinib-letrozole combination in post-menopausal patients with HR + HER2+ metastatic breast cancer [11]. A high pathological complete response (pCR, 21\%; pathologic response rate, 54\%) was also observed with add-on letrozole (+ luteinizing hormone-releasing hormone $[\mathrm{LHRH}]$ agonist in premenopausal women) to a 12-week trastuzumab-lapatinib combination in patients with estrogen receptor (ER) positive $(E R+)$ HER2+ breast cancer in a phase 2 study [12]. Similarly, the trastuzumab-anastrozole combination showed prolonged progression-free survival (PFS; median, 4.8 months) in patients with HER $2+\mathrm{HR}+$ metastatic breast cancer in the phase 3 TAnDEM study [13]. In the PERTAIN study, the PFS was 18.9 months/15.8 months in patients with HER2+ $\mathrm{HR}+$ metastatic breast cancer/locally advanced breast cancer who received trastuzumab + aromatase inhibitor (AI) with/ without pertuzumab, respectively [14]. A systematic review concluded that treatment with lapatinib/trastuzumab + AI was clinically more effective than AI monotherapy in patients with HR + HER2+ breast cancer [15]. Thus, concomitant HT is expected to show additional efficacy due to dual action, in ER+ HER2+ breast cancer.

Furthermore, among the common breast cancer subtypes, higher pCR rates were observed in ER-negative (ER-), luminal B HER2-negative (HER2-), nonluminal HER2+, and triple-negative (ER-, progesterone receptor $[\mathrm{PgR}]$-negative, and HER2-) disease [16].

Therefore, the key considerations that remain are improving pCR rates in luminal HER2+ disease, which is less sensitive to the combination of chemotherapy and antiHER 2+ agents, and minimizing treatment-related toxicity without reducing efficacy in terms of pCR rates. This randomized, phase 2, 3-arm study was designed to compare docetaxel + carboplatin + trastuzumab + pertuzumab (TCbHP), TCbHP followed by T-DM1+P, and T-DM1+P for treating HER2+ primary breast cancer patients as a Japan Breast 
Cancer Research Group (JBCRG) association study-20, Neopeaks. In the T-DM1+P arm, we personalized the treatment after confirming tumor shrinkage and responders continued on the same treatment, whereas nonresponders were switched to a different type of anthracycline-based regimen.

\section{Materials and methods}

\section{Study design}

This randomized, phase 2, open-label, 3-arm study enrolled patients between August 2014 and February 2016 at 17 institutions/centers across Japan. Eligible patients were randomized in a 1:1:2 ratio into 3 treatment groups: group A received 6 cycles of TCbHP; group B received 4 cycles of TCbHP followed by 4 cycles of T-DM1+P; and group $C$ (the response-guided regimen) received 4 cycles of T-DM1+P followed by 2 cycles of T-DM1+P among responders (subgroup $\mathrm{C} 1$ ) or switched to 4 cycles of 5-fluorouracil + epirubicin + cyclophosphamide (FEC) among nonresponders (subgroup C2) (Online Resource 1). Responders were defined as patients with $\geq 30 \%$ decrease in tumor size by magnetic resonance imaging (MRI) and with a Ki67 level of $\leq 10 \%$ or absence of cancer cells in core needle biopsy. Patients received the assigned regimens on day 1 of each 3-week cycle. Minimization method was used, and treatment allocation adjustment factors were ER status, menopausal status, T1-T2/T3, N0/ $\mathrm{N} 1$, and institution. Breast surgery was performed within 10 weeks of neoadjuvant therapy completion. The study was conducted in accordance with the Declaration of Helsinki and Guidelines for Good Clinical Practice. The study was approved by the Institutional Review Board of each participating institution. Written informed consent was obtained from all patients.

\section{Patients}

Key inclusion criteria were women aged $\geq 20$ and $\leq 70$ years with Eastern Cooperative Oncology Group performance status $0-1$, histologically confirmed primary invasive breast cancer (cT1 c-cT3, cN0-cN1, cM0), targeted lesion size $\leq 7 \mathrm{~cm}$ by MRI or ultrasound imaging, and appropriately maintained organ function. HER2 expression was determined using immunohistochemistry (IHC) and in situ hybridization (fluorescence in situ hybridization [FISH] and dual in situ hybridization [DISH] assays) by central laboratory testing at the Department of Diagnostic Pathology, Kyoto University Hospital, in accordance with the recommendations for HER2 testing in breast cancer by the American Society of Clinical
Oncology/College of American Pathologists in the 2013 updated clinical practice guidelines [17]. ER expression status by IHC and Ki67 index was also investigated mandatorily at the central laboratory after obtaining written informed consent. Patients with bilateral breast cancer, axillary lymph node dissection before neoadjuvant chemotherapy, incision/excision biopsy of the primary lesion or axillary lymph node(s), multiple primary cancers, and/or grade $\geq 2$ peripheral sensory neuropathy were excluded.

\section{Treatment}

Details of dose schedule, concurrent HT, and discontinuation or treatment suspension criteria are described in Online Resource 2. Patients with ER+ were treated with concurrent HT along with T-DM1+P; pre-menopausal patients received an LHRH analog with tamoxifen, whereas post-menopausal patients received letrozole. Breast surgery (total mastectomy, partial mastectomy $[\mathrm{Bp}]$, or quadrantectomy $[\mathrm{Bq}]$ ) was performed within 10 weeks of neoadjuvant therapy completion at the investigator's discretion. Immediate breast reconstruction was allowed for mastectomy cases.

Post-operative adjuvant therapy was performed by investigator choice according to the clinical guidelines. One-year treatment was recommended for trastuzumab (including the neoadjuvant therapy or T-DM1 treatment period). When residual, invasive cancer was pathologically observed, addition of appropriate chemotherapy (e.g., anthracyclines) was recommended. In patients with ER+, HT was recommended for $\geq 5$ years. Local radiation therapy was performed when deemed necessary (Online Resource 2).

\section{Endpoints}

Primary endpoint was pCR (comprehensive pCR [CpCR] ypN0 [ypT0-TisypN0], including residual ductal carcinoma in situ) rate by central histopathological review. Exploratory analyses assessed pCR rates based on ER status. Secondary endpoints were (1) CpCR defined as noninvasive cancer or in situ tumor residuals (strict $\mathrm{pCR}[\mathrm{SpCR}]+$ invasive pCR [pCRinv]); (2) SpCR (histological absence of tumor [grade 3] with pathological evidence of cancer prior to treatment + noninvasive cancer or in situ tumor residuals [pCRinv]); (3) Quasi pCR (QpCR) defined as limited number of tumor cells present in the removed breast tissue (grade 2b) [18]; and (4) QpCR + ypN0. Other endpoints included overall response rate (ORR), clinical complete response (cCR) assessed by MRI/positron emission tomographycomputed tomography (PET-CT), tumor shrinkage assessed by MRI/PET-CT at cycle 4, breast conservation rate (breastconserving surgery [BCS; Bp or Bq] with clear margin), 
breast conservation rate in patients who shifted from preplanned mastectomy to BCS, disease-free survival (DFS), overall survival (OS), and safety using the Medical Dictionary for Regulatory Activities/Japanese (MedDRA/J) v18.1 and graded per the National Cancer Institute Common Terminology Criteria for Adverse Events (NCI CTCAE) v4.03.

\section{Statistical analysis}

For groups A and B, a total of 50 patients each were set to yield $95 \%$ CIs of $45.2-73.6 \%$ and $61.8-86.9 \%$, respectively, assuming true $\mathrm{pCR}$ rates of $60 \%$ and $75 \%$, respectively. For group C, a total of 100 patients were set to yield a $95 \%$ CI of $65.3-83.1 \%$, assuming a true pCR rate of $75 \%$. For efficacy and safety endpoints, point estimates and 95\% CIs were calculated or $\chi^{2}$ test was used (significance level 0.05 ) to compare across groups. $\mathrm{pCR}$ rates in $\mathrm{ER}+/-$ patients were compared across groups. In an exploratory analysis, pCR rates stratified by HER 2 expression status (IHC3+ [strong] or IHC2+ [equivocal] plus DISH+ [mild]) were compared within the group. DFS and OS curves were estimated using the Kaplan-Meier method.

\section{Results}

\section{Patient disposition and baseline characteristics}

Of the 236 patients primarily enrolled, 204 were randomized to groups $\mathrm{A}(n=51), \mathrm{B}(n=52)$, and $\mathrm{C}(n=101)$ (Fig. 1); 32 were excluded, most commonly due to HER2 negative status $(n=16)$ by central laboratory testing. Within group C, 80
(79.2\%) patients continued T-DM1+P (subgroup C1) after a favorable response, whereas $21(20.8 \%)$ nonresponders were switched to FEC (subgroup C2; Online Resource 3). Overall, 49 patients each in groups $\mathrm{A}$ and $\mathrm{B}$ and 96 patients in group C completed the protocol-specified therapy (Fig. 1). Dose reductions were required for docetaxel (relative dose intensity [RDI], 94.9\%) in 10 (19.6\%) patients and for carboplatin (RDI, 93.7\%) in $12(23.5 \%)$ patients in group A, for docetaxel (RDI, 95.3\%) and carboplatin (RDI, 95.0\%) in $9(17.3 \%)$ patients each and T-DM1 (RDI, 97.9\%) in 8 (15.4\%) patients in group B, and for T-DM1 (RDI, 97.6\%) in $11(10.9 \%)$ patients in group C. Patients were generally well matched across treatment groups with a median (range) age of 53 (25-70) years and tumor size of $26(11-70) \mathrm{mm}$. Overall, 118 (57.8\%) patients were ER+ and 94 (46.1\%) were post-menopausal (Table 1).

\section{Post-operative adjuvant therapy}

Overall, post-operative adjuvant chemotherapy was administered in 36/204 (17.6\%) patients (with pCR, 5/124 [4.0\%]; without pCR, 31/80 [38.8\%]), with $28(77.8 \%)$ of them receiving an anthracycline-containing regimen. By treatment groups, 10/103 (anthracyclines in 9/10) patients in groups A and B and 26/101 (anthracyclines in 19/26) in group C were administered post-operative therapy. Overall, the most common post-operative therapy administered was trastuzumab (98\%, 200/204), and concomitant HT (54.9\%, 112/204) based on histological examination of tumor tissue by core needle biopsy or residual disease on surgical specimen.

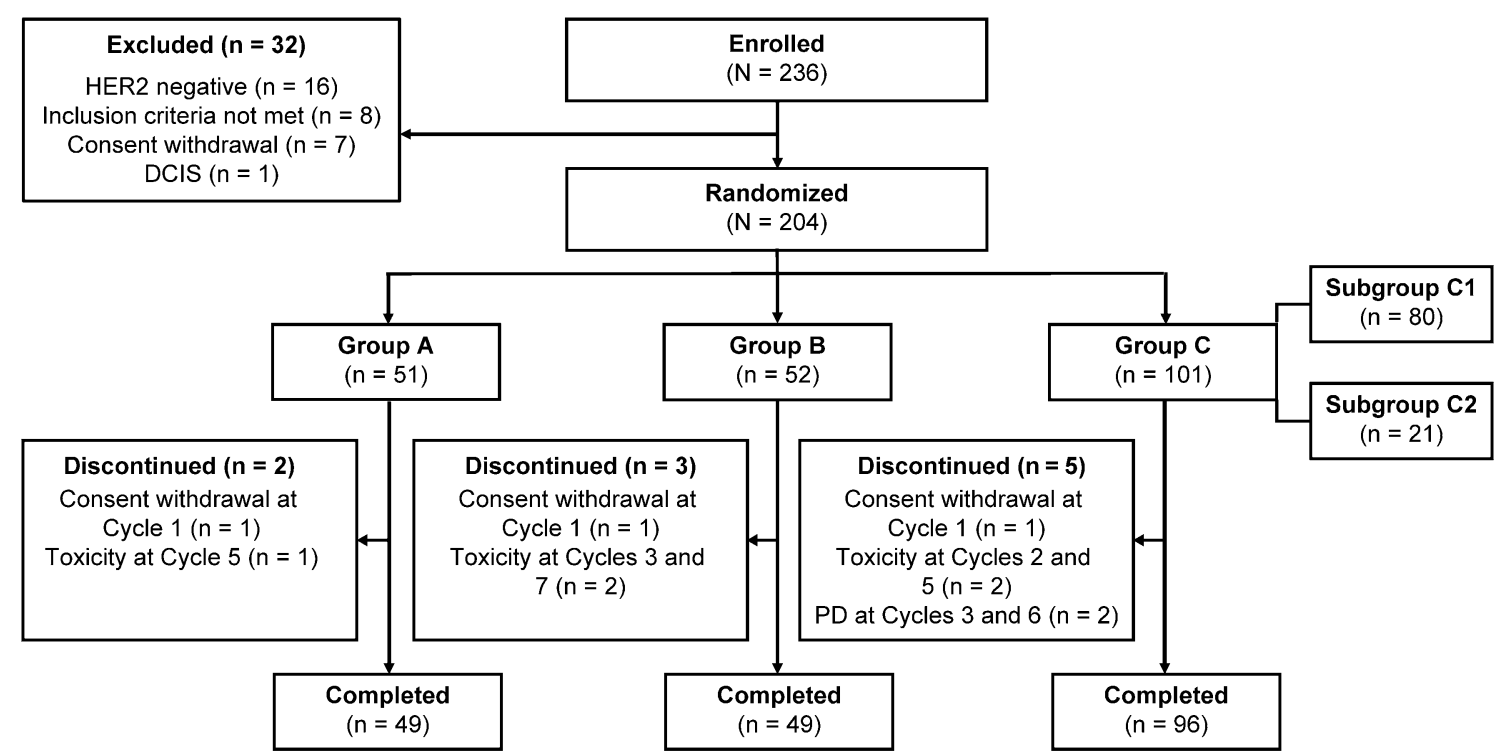

Fig. 1 Patient disposition. PD progressive disease, DCIS ductal carcinoma in situ, HER2 human epidermal growth factor receptor 2 
Table 1 Patient demographics and baseline characteristics

\begin{tabular}{|c|c|c|c|c|c|c|}
\hline Variable & $\begin{array}{l}\text { Overall } \\
(N=204)\end{array}$ & $\begin{array}{l}\text { Group A } \\
(n=51)\end{array}$ & $\begin{array}{l}\text { Group B } \\
(n=52)\end{array}$ & $\begin{array}{l}\text { Group C } \\
(n=101)\end{array}$ & $\begin{array}{l}\text { Subgroup C1 } \\
(n=80)\end{array}$ & $\begin{array}{l}\text { Subgroup C2 } \\
(n=21)\end{array}$ \\
\hline \multicolumn{7}{|l|}{ Age, years } \\
\hline Median (range) & $53.0(25-70)$ & $53.0(28-70)$ & $53.0(29-69)$ & $52.0(25-70)$ & $51.5(25-70)$ & $53.0(40-67)$ \\
\hline \multicolumn{7}{|c|}{ Menopausal status, $n(\%)$} \\
\hline Post-menopause & $94(46.1)$ & $23(45.1)$ & $23(44.2)$ & $48(47.5)$ & $39(48.8)$ & $9(42.9)$ \\
\hline Pre-menopause & $110(53.9)$ & $28(54.9)$ & $29(55.8)$ & $53(52.5)$ & $41(51.3)$ & $12(57.1)$ \\
\hline \multicolumn{7}{|c|}{ ECOG performance status, $n(\%)$} \\
\hline 0 & $203(99.5)$ & $51(100.0)$ & $52(100.0)$ & $100(99.0)$ & $79(98.8)$ & $21(100.0)$ \\
\hline 1 & $1(0.5)$ & $0(0.0)$ & $0(0.0)$ & $1(1.0)$ & $1(1.3)$ & $0(0.0)$ \\
\hline \multicolumn{7}{|c|}{ T stage (primary tumor), $n(\%)$} \\
\hline $\mathrm{T} 1 \mathrm{c}$ & $44(21.6)$ & $11(21.6)$ & $13(25.0)$ & $20(19.8)$ & $14(17.5)$ & $6(28.6)$ \\
\hline $\mathrm{T} 2$ & $144(70.6)$ & $37(72.5)$ & $35(67.3)$ & $72(71.3)$ & $58(72.5)$ & $14(66.7)$ \\
\hline $\mathrm{T} 3$ & $16(7.8)$ & $3(5.9)$ & $4(7.7)$ & $9(8.9)$ & $8(10.0)$ & $1(4.8)$ \\
\hline \multicolumn{7}{|c|}{ Tumor size by MRI/PET-CT, mm } \\
\hline Median (range) & $26.0(11-70)$ & $27.0(11-58)$ & $25.5(12-56)$ & $27.0(11-70)$ & $27.0(11-70)$ & $27.0(12-51)$ \\
\hline \multicolumn{7}{|l|}{$\mathrm{N}$ stage, $n(\%)$} \\
\hline N0 & $129(63.2)$ & $34(66.7)$ & $31(59.6)$ & $64(63.4)$ & $49(61.3)$ & $15(71.4)$ \\
\hline N1 & 75 (36.8) & $17(33.3)$ & $21(40.4)$ & $37(36.6)$ & $31(38.8)$ & $6(28.6)$ \\
\hline \multicolumn{7}{|l|}{ HER2 status, $n(\%)$} \\
\hline IHC3+ & $177(86.8)$ & $45(88.2)$ & $45(86.5)$ & $87(86.1)$ & $70(87.5)$ & $17(81.0)$ \\
\hline IHC $2+/ \mathrm{CISH}+$ & $27(13.2)$ & $6(11.8)$ & $7(13.5)$ & $14(13.9)$ & $10(12.5)$ & $4(19.0)$ \\
\hline \multicolumn{7}{|l|}{ ER status, $n(\%)$} \\
\hline Positive & $118(57.8)$ & $30(58.8)$ & $29(55.8)$ & $59(58.4)$ & $44(55.0)$ & $15(71.4)$ \\
\hline Negative & $86(42.2)$ & $21(41.2)$ & $23(44.2)$ & $42(41.6)$ & $36(45.0)$ & $6(28.6)$ \\
\hline \multicolumn{7}{|l|}{ Ki67 index, $n(\%)$} \\
\hline$<10 \%$ & $10(4.9)$ & $2(3.9)$ & $2(3.8)$ & $6(5.9)$ & $4(5.0)$ & $2(9.5)$ \\
\hline $10 \%$ to $<20 \%$ & $37(18.1)$ & $11(21.6)$ & $7(13.5)$ & $19(18.8)$ & $14(17.5)$ & $5(23.8)$ \\
\hline $20 \%$ to $<30 \%$ & $49(24.0)$ & $10(19.6)$ & $17(32.7)$ & $22(21.8)$ & $19(23.8)$ & $3(14.3)$ \\
\hline $30 \%$ to $<50 \%$ & $65(31.9)$ & $16(31.4)$ & $11(21.2)$ & $38(37.6)$ & $32(40.0)$ & $6(28.6)$ \\
\hline$\geq 50 \%$ & $43(21.1)$ & $12(23.5)$ & $15(28.8)$ & $16(15.8)$ & $11(13.8)$ & $5(23.8)$ \\
\hline \multicolumn{7}{|c|}{ Planned surgical procedure, $n(\%)$} \\
\hline $\mathrm{Bt}$ & $128(62.7)$ & $33(64.7)$ & $31(59.6)$ & $64(63.4)$ & $50(62.5)$ & $14(66.7)$ \\
\hline $\mathrm{Bp} / \mathrm{Bq}$ & $76(37.3)$ & $18(35.3)$ & $21(40.4)$ & $37(36.6)$ & $30(37.5)$ & $7(33.3)$ \\
\hline
\end{tabular}

$B p$ partial mastectomy, $B q$ quadrantectomy, $B t$ total mastectomy, $C I S H$ chromogenic in situ hybridization, ECOG Eastern Cooperative Oncology Group, ER estrogen receptor, HER2 human epidermal growth factor receptor 2, IHC immunohistochemistry, IHC2+ equivocal for HER2 protein expression (circumferential membrane staining that is incomplete, weak, or moderate within $>10 \%$ of the invasive tumor cells or complete and circumferential intense membrane staining within $\leq 10 \%$ of invasive tumor cells), IHC3+ positive HER 2 expression (circumferential membrane staining that is complete, intense, and in $>10 \%$ of invasive tumor cells), MRI magnetic resonance imaging, PET-CT positron emission tomography-computed tomography

\section{Pathological complete response}

pCR rate was numerically higher in group B (71.2\%) than in groups A (56.9\%) and C (57.4\%); all between-group comparisons were not significant $(P<0.05$, by chi-square test) except group B vs group C2 $(P=0.0086)$, and group $\mathrm{C} 1 \mathrm{vs}$ group $\mathrm{C} 2(P=0.0441)$. The rates were comparable between groups $\mathrm{A}$ and $\mathrm{C}$ and subgroup $\mathrm{C} 1$. pCR rate was lowest $(38.1 \%)$ in the $\mathrm{T}-\mathrm{DM} 1+\mathrm{P}$ nonresponder subgroup $\mathrm{C} 2$ who were switched to FEC (Fig. 2a). Results of the exploratory analysis showed that the $\mathrm{pCR}$ rate was significantly higher in group $\mathrm{B}$ than in groups $\mathrm{A}(P=0.047)$ and $\mathrm{C}(P=0.013)$ in patients with $\mathrm{ER}+$ but was comparable in patients with ER- (Fig. 2b). There were no significant differences between the groups for other secondary endpoints of pathological response rate (Table 2). Results of the exploratory analysis showed that $\mathrm{pCR}$ rates were significantly higher $(P<0.01)$ in patients with strong HER2 expression (HER2IHC $3+$ ) than those with mild HER2 expression (HER2IHC $2+$, chromogenic in situ hybridization [CISH]-positive) 
Fig. 2 pCR rate in a all patient groups, b ER+/- patients, and c patients with strong/mild HER2 expression. DISH dual in situ hybridization, $E R$ estrogen receptor, HER2 human epidermal growth factor receptor 2 , IHC immunohistochemistry, $I H C 2+$ equivocal for HER2 protein expression (circumferential membrane staining that is incomplete, weak, or moderate within $>10 \%$ of invasive tumor cells or complete and circumferential intense membrane staining within $\leq 10 \%$ of invasive tumor cells); $I H C 3+$ positive HER2 expression (circumferential membrane staining that is complete, intense, and in $>10 \%$ of invasive tumor cells), $p C R$ pathological complete response a

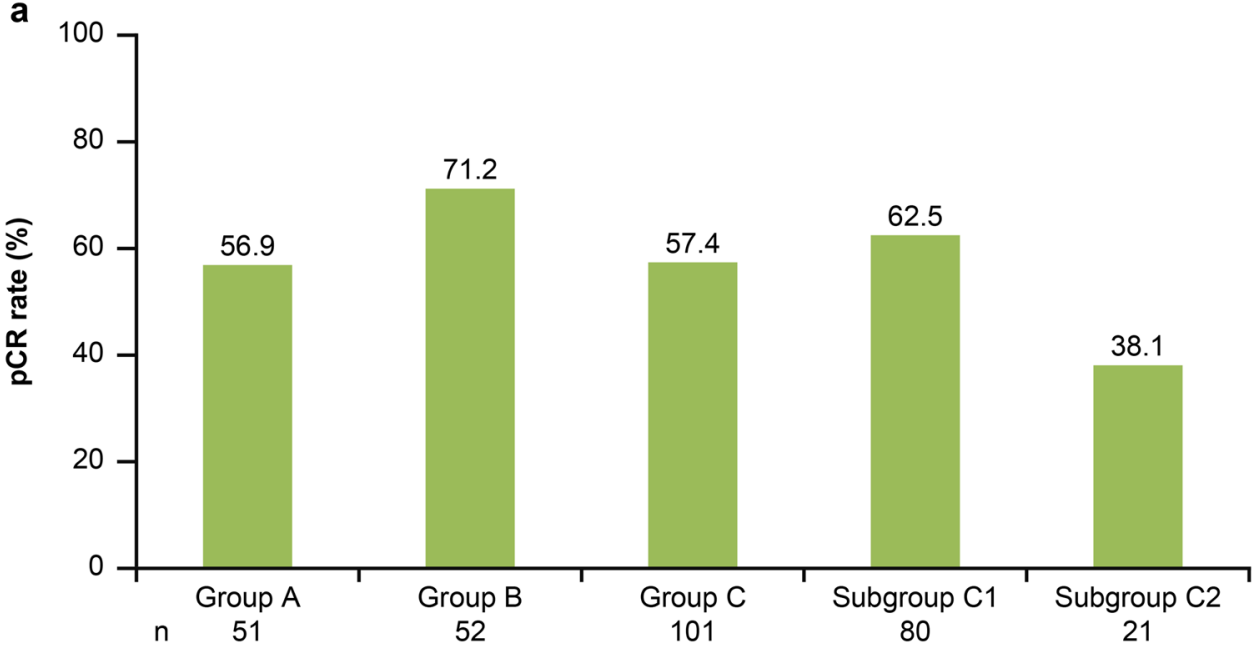

b

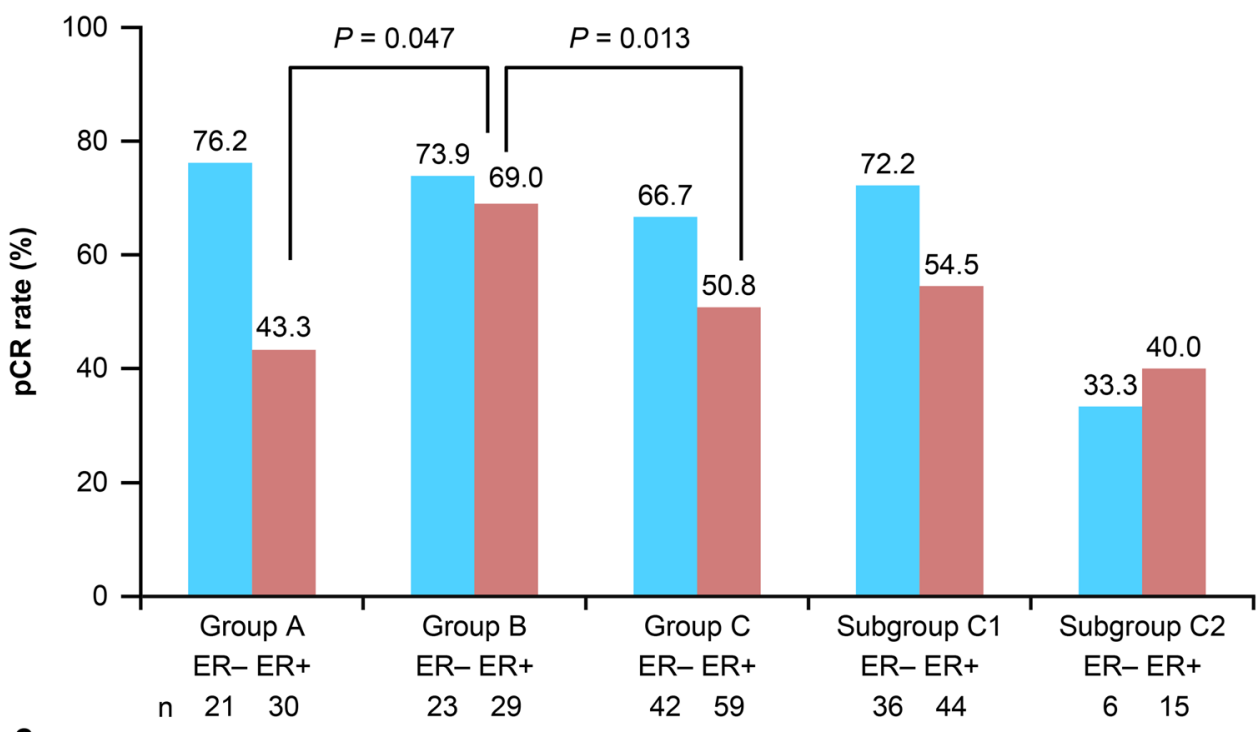

C

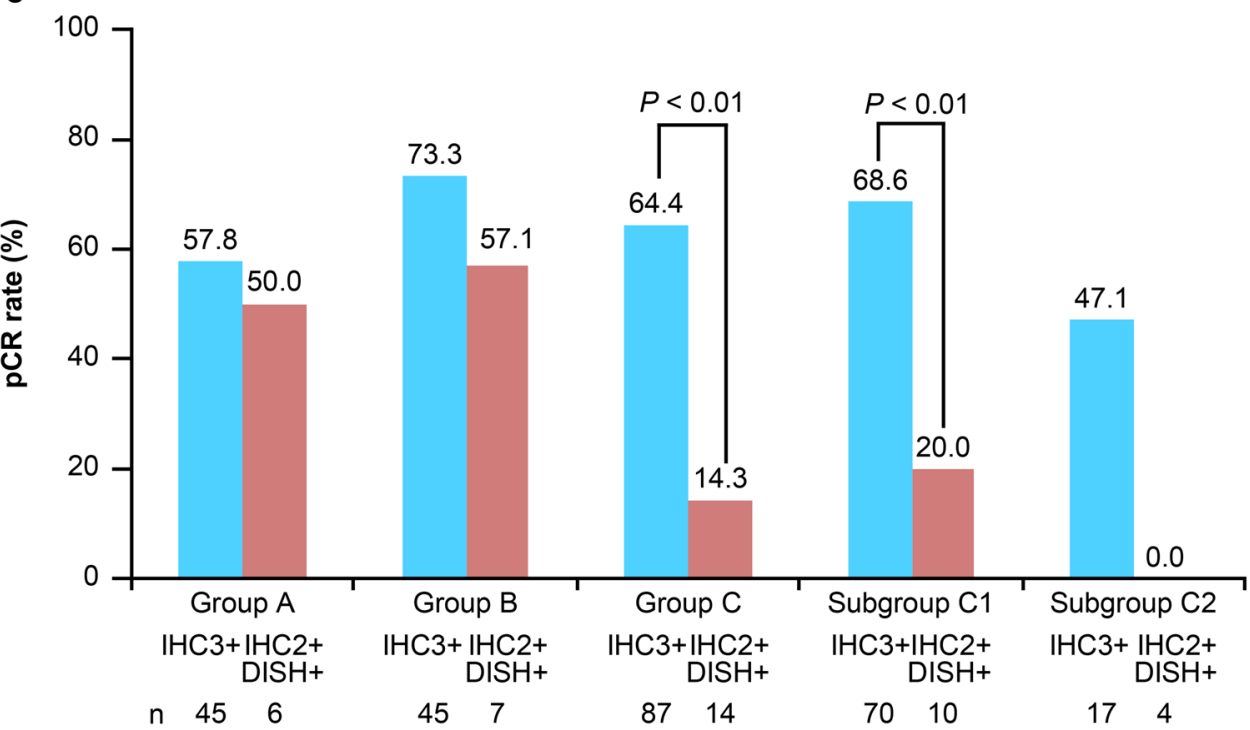


Table 2 Pathological response rate (full analysis set, $N=204$ ), clinical response rate, and breast conservation rate

\begin{tabular}{llllll}
\hline Variable & $\begin{array}{l}\text { Group A } \\
(n=51)\end{array}$ & $\begin{array}{l}\text { Group B } \\
(n=52)\end{array}$ & $\begin{array}{l}\text { Group C } \\
(n=101)\end{array}$ & $\begin{array}{l}\text { Subgroup C1 } \\
(n=80)\end{array}$ & $\begin{array}{l}\text { Subgroup C2 } \\
(n=21)\end{array}$ \\
\hline CpCR $^{\mathrm{a}}$ & $29(56.9 \%)$ & $37(71.2 \%)$ & $58(57.4 \%)$ & $50(62.5 \%)$ & $8(38.1 \%)$ \\
$95 \%$ CI & $(42.2,70.7)$ & $(56.9,82.9)$ & $(47.2,67.2)$ & $(51.0,73.1)$ & $(18.1,61.6)$ \\
SpCR $^{\mathrm{a}}$ & $22(43.1 \%)$ & $30(57.7 \%)$ & $43(42.6 \%)$ & $36(45.0 \%)$ & $7(33.3 \%)$ \\
$95 \%$ CI & $(29.3,57.8)$ & $(43.2,71.3)$ & $(32.8,52.8)$ & $(33.8,56.5)$ & $(14.6,57.0)$ \\
QpCR & $39(76.5 \%)$ & $43(82.7 \%)$ & $68(67.3 \%)$ & $58(72.5 \%)$ & $10(47.6 \%)$ \\
$95 \%$ CI & $(62.5,87.2)$ & $(69.7,91.8)$ & $(57.3,76.3)$ & $(61.4,81.9)$ & $(25.7,70.2)$ \\
QpCR and ypN0 & $38(74.5 \%)$ & $40(76.9 \%)$ & $68(67.3 \%)$ & $58(72.5 \%)$ & $10(47.6 \%)$ \\
$95 \%$ CI & $(60.4,85.7)$ & $(63.2,87.5)$ & $(57.3,76.3)$ & $(61.4,81.9)$ & $(25.7,70.2)$ \\
ORR (by investigator's assessment) & $49(96.1 \%)$ & $45(86.5 \%)$ & $89(88.1 \%)$ & $71(88.8 \%)$ & $18(85.7 \%)$ \\
$95 \%$ CI & $(86.5,99.5)$ & $(74.2,94.4)$ & $(80.2,93.7)$ & $(79.7,94.7)$ & $(63.7,97.0)$ \\
cCR rate (by MRI/PET-CT) & $24(47.1 \%)$ & $27(51.9 \%)$ & $39(38.6 \%)$ & $31(38.8 \%)$ & $8(38.1 \%)$ \\
95\% CI & $(32.9,61.5)$ & $(37.6,66.0)$ & $(29.1,48.8)$ & $(28.1,50.3)$ & $(18.1,61.6)$ \\
Breast conservation rate, $n / N^{\mathrm{b}}(\%)$ & $26 / 50(52.0 \%)$ & $27 / 52(51.9 \%)$ & $51 / 100(51.0 \%)$ & $43 / 79(54.4 \%)$ & $8 / 21(38.1 \%)$ \\
Breast conservation rate in patients who had $)$ & $11 / 32(34.4 \%)$ & $12 / 31(38.7 \%)$ & $20 / 63(31.7 \%)$ & $18 / 49(36.7 \%)$ & $2 / 14(14.3 \%)$ \\
pre-planned mastectomy, $n / N^{\mathrm{b}}(\%)$ & & & & \\
\hline
\end{tabular}

$B p$ partial mastectomy, $B q$ quadrantectomy, $c C R$ complete clinical response, $C I$ confidence interval, $C p C R$ comprehensive pCR, $p C R$ pathological complete response, MRI magnetic resonance imaging, ORR overall response rate, PET-CT positron emission tomography-computed tomography, $Q p C R$ quasi $\mathrm{pCR}, S p C R$ strict $\mathrm{pCR}$

${ }^{a}$ There were no patients with lymph node metastasis who achieved CpCR or SpCR. The rate of CpCR and CpCRypN0 was identical as was the rate of SpCR and SpCRypNO

${ }^{\mathrm{b}} \mathrm{Patients}$ who underwent $\mathrm{Bp}$ or $\mathrm{Bq}$ and had a negative margin were defined as successful breast conservation

in group C (56/87 [64.4\%] versus $2 / 14[14.3 \%])$ and subgroup C1 (48/70 [68.6\%] versus $2 / 10$ [20.0\%]), respectively (Fig. 2c).

\section{Clinical response}

ORR was high and comparable (86-96\%) among groups (Table 2), and disease progression was observed in 2 patients in group C (assessed by Response Evaluation Criteria In Solid Tumors [RECIST] v1.1). cCR rate was comparable between groups A (47\%) and B (52\%), but marginally lower in group $\mathrm{C}(39 \%)$ and did not differ in the response-guided subgroups C1 (39\%) and C2 (38\%) (Table 2). There was a decrease in tumor size from baseline at cycle 4 in all patients, except 1 each in group A and subgroup $\mathrm{C} 1$ (pCR achieved), and subgroup C2 (pCR not achieved) (Online Resource 4).

\section{Breast conservation rate}

Breast conservation was achieved in approximately half (51-54\%) of the patients in groups A, B, and C and subgroup $\mathrm{C} 1$; success rate was lower in subgroup C2 (38\%). Similarly, among patients who underwent BCS instead of planned mastectomy, breast conservation success rate was higher in groups A, B, and C and subgroup C1 (32-39\%) than subgroup $\mathrm{C} 2$ (14\%) (Table 2).

\section{Disease-free survival and overall survival}

At the median follow-up of 1064 days (range 705-1541 days), 3, 3, and 5 events of recurrence had been reported in groups $\mathrm{A}, \mathrm{B}$, and $\mathrm{C}$, respectively, and 2, 2, and 3 patients in groups $\mathrm{A}, \mathrm{B}$, and $\mathrm{C}$, respectively, had events of distant recurrence. Only 1 patient in group A had died due to breast cancer. The rate of DFS at 3 years was $94.3 \%$ in group A, $96.2 \%$ in group B, and $94.0 \%$ in group C. The rate of OS at 3 years was $99.2 \%$ among all patients.

\section{Safety}

Treatment-related adverse events (AEs) by treatment group ( $\geq 10 \%$ incidence) are presented in Online Resource 5. Most commonly reported drug-related AEs $(\geq 10 \%$ incidence and $\geq 10 \%$ difference between group A and subgroup C1) were alopecia ( $94.1 \%$ versus $5 \%$ ), diarrhea ( $86.3 \%$ versus $32.5 \%)$, decrease in white blood cell count $(86.3 \%$ versus $8.8 \%$ ), and neutropenia ( $84.3 \%$ versus $20 \%$ ) (Table 3 ). Most commonly reported drug-related AEs $(\geq 10 \%$ incidence and $\geq 10 \%$ difference between group B and subgroup C1) were alopecia $(86.5 \%$ versus $5 \%)$, neutropenia $(76.9 \%$ versus $20 \%$ ), and nausea (75\% versus $50 \%$ ). Grade $3 / 4$ AEs were significantly less frequent in subgroup C1 $(33.8 \%)$ than group A $(84.3 \%)$, group B $(76.9 \%)$, or subgroup C2 
Table 3 Drug-related adverse events ( $\geq 10 \%$ incidence and $\geq 10 \%$ difference between group A and subgroup C1 or group B and subgroup C1)

\begin{tabular}{|c|c|c|c|c|c|}
\hline Drug-related adverse event & $\begin{array}{l}\text { Overall, } n(\%) \\
(n=204)\end{array}$ & $\begin{array}{l}\text { Group A, } n(\%) \\
(n=51)\end{array}$ & $\begin{array}{l}\text { Group B, } n(\%) \\
(n=52)\end{array}$ & $\begin{array}{l}\text { Subgroup C1, } n(\%) \\
(n=80)\end{array}$ & $\begin{array}{l}\text { Subgroup C2, } n(\%) \\
(n=21)\end{array}$ \\
\hline \multicolumn{6}{|l|}{ Blood and lymphatic system disorders } \\
\hline Neutropenia & $107(52.5)$ & $43(84.3)$ & $40(76.9)$ & $16(20.0)$ & $8(38.1)$ \\
\hline Anemia & $72(35.3)$ & $27(52.9)$ & $31(59.6)$ & $8(10.0)$ & $6(28.6)$ \\
\hline Febrile neutropenia & $26(12.7)$ & $11(21.6)$ & $8(15.4)$ & $0(0.0)$ & $7(33.3)$ \\
\hline \multicolumn{6}{|l|}{ Metabolism and nutrition disorders } \\
\hline Decreased appetite & $74(36.3)$ & $25(49.0)$ & $21(40.4)$ & $19(23.8)$ & $9(42.9)$ \\
\hline \multicolumn{6}{|l|}{ Psychiatric disorders } \\
\hline Insomnia & $13(6.4)$ & $7(13.7)$ & $3(5.8)$ & $2(2.5)$ & $1(4.8)$ \\
\hline \multicolumn{6}{|l|}{ Nervous system disorders } \\
\hline Dysgeusia & $100(49.0)$ & $35(68.6)$ & $38(73.1)$ & $16(20.0)$ & $11(52.4)$ \\
\hline Neuropathy peripheral & $64(31.4)$ & $25(49.0)$ & $28(53.8)$ & $8(10.0)$ & $3(14.3)$ \\
\hline \multicolumn{6}{|l|}{ Gastrointestinal disorders } \\
\hline Nausea & $136(66.7)$ & $39(76.5)$ & $39(75.0)$ & $40(50.0)$ & $18(85.7)$ \\
\hline Diarrhea & $119(58.3)$ & $44(86.3)$ & $38(73.1)$ & $26(32.5)$ & $11(52.4)$ \\
\hline Stomatitis & $111(54.4)$ & $40(78.4)$ & $31(59.6)$ & $25(31.3)$ & $15(71.4)$ \\
\hline Constipation & $61(29.9)$ & $24(47.1)$ & $20(38.5)$ & $8(10.0)$ & $9(42.9)$ \\
\hline Vomiting & $56(27.5)$ & $21(41.2)$ & $21(40.4)$ & $5(6.3)$ & $9(42.9)$ \\
\hline Abdominal pain upper & $29(14.2)$ & $13(25.5)$ & $7(13.5)$ & $5(6.3)$ & $4(19.0)$ \\
\hline \multicolumn{6}{|l|}{ Skin and subcutaneous tissue disorders } \\
\hline Alopecia & $114(55.9)$ & $48(94.1)$ & $45(86.5)$ & $4(5.0)$ & $17(81.0)$ \\
\hline Dermatitis acneiform & $44(21.6)$ & $17(33.3)$ & $13(25.0)$ & $11(13.8)$ & $3(14.3)$ \\
\hline Palmar-plantar erythrodysesthesia syndrome & $28(13.7)$ & $13(25.5)$ & $14(26.9)$ & $0(0.0)$ & $1(4.8)$ \\
\hline Nail discoloration & $22(10.8)$ & $9(17.6)$ & $13(25.0)$ & $0(0.0)$ & $0(0.0)$ \\
\hline Nail disorder & $17(8.3)$ & $7(13.7)$ & $7(13.5)$ & $1(1.3)$ & $2(9.5)$ \\
\hline \multicolumn{6}{|l|}{ Musculoskeletal and connective tissue disorders } \\
\hline Myalgia & $31(15.2)$ & $9(17.6)$ & $16(30.8)$ & $4(5.0)$ & $2(9.5)$ \\
\hline Arthralgia & $30(14.7)$ & $4(7.8)$ & $14(26.9)$ & $8(10.0)$ & $4(19.0)$ \\
\hline \multicolumn{6}{|l|}{ General disorders and administration site conditions } \\
\hline Malaise & $86(42.2)$ & $27(52.9)$ & $25(48.1)$ & $22(27.5)$ & $12(57.1)$ \\
\hline Pyrexia & $41(20.1)$ & $15(29.4)$ & $12(23.1)$ & $5(6.3)$ & $9(42.9)$ \\
\hline Edema peripheral & $31(15.2)$ & $17(33.3)$ & $12(23.1)$ & $0(0.0)$ & $2(9.5)$ \\
\hline Fatigue & $24(11.8)$ & $8(15.7)$ & $7(13.5)$ & $4(5.0)$ & $5(23.8)$ \\
\hline Edema & $11(5.4)$ & $7(13.7)$ & $4(7.7)$ & $0(0.0)$ & $0(0.0)$ \\
\hline \multicolumn{6}{|l|}{ Investigations } \\
\hline Platelet count decreased & $114(55.9)$ & $12(23.5)$ & $30(57.7)$ & $55(68.8)$ & $17(81.0)$ \\
\hline Alanine aminotransferase increased & $98(48.0)$ & $20(39.2)$ & $23(44.2)$ & $42(52.5)$ & $13(61.9)$ \\
\hline White blood cell count decreased & $97(47.5)$ & $44(86.3)$ & $38(73.1)$ & $7(8.8)$ & $8(38.1)$ \\
\hline Aspartate aminotransferase increased & $91(44.6)$ & $15(29.4)$ & $22(42.3)$ & $41(51.3)$ & $13(61.9)$ \\
\hline Weight decreased & $20(9.8)$ & $8(15.7)$ & $9(17.3)$ & $0(0.0)$ & $3(14.3)$ \\
\hline Blood alkaline phosphatase increased & $19(9.3)$ & $1(2.0)$ & $4(7.7)$ & $13(16.3)$ & $1(4.8)$ \\
\hline \multicolumn{6}{|l|}{ Injury, poisoning, and procedural complications } \\
\hline Infusion related reaction & $89(43.6)$ & $10(19.6)$ & $21(40.4)$ & $45(56.3)$ & $13(61.9)$ \\
\hline
\end{tabular}

(76.2\%; all $P<0.001)$. Grade 3/4 neutropenia, febrile neutropenia, and leukopenia were reported less often in subgroup C1 than groups A or B, or subgroup C2 (Online Resource 6). No unexpected drug-related AEs were reported. Drugrelated alopecia and febrile neutropenia were less frequent in subgroup $\mathrm{C} 1(5.0 \%, 0 \%)$ than group A $(94.1 \%, 21.6 \%)$, group B $(86.5 \%, 15.4 \%)$, or subgroup C2 $(81 \%, 33.3 \%)$. No treatment discontinuations or deaths due to AEs were reported. 


\section{Discussion}

Results of this study showed that the ypT0/is and ypN0 rate was numerically higher with the $\mathrm{TCbHP} \rightarrow \mathrm{T}-\mathrm{DM} 1+\mathrm{P}$ regimen (71\%; group B) than with the standard TCbHP regimen (57\%; group A) and the T-DM1+P regimen (57\%; group C). As expected, in group $\mathrm{C}$, the rate was higher among responders who continued T-DM1+P (63\%; subgroup $\mathrm{C} 1)$ than in nonresponders who switched to FEC (38\%; subgroup C2). Notably, the pCR rate (57.4\%) after the initial 4 cycles of T-DM1+P (group C) was equivalent to that with the standard TCbHP regimen (57\%). Of note, the pCR rate in the $\mathrm{ER}+$ subgroup of patients was significantly higher in group B (69\%) than groups A (43.3\%) and C (50.8\%), but was comparable in ER- subgroups of patients (66.7-76.2\%).

The overall rate of OS was $99.2 \%$ and rate of DFS was $\geq 94 \%$ in all groups at 3 years in our study, which are reflective of anthracycline-free post-operative therapy in $91.2 \%$ of patients in groups A and B. In group C, post-operative anthracyclines were used in approximately $60 \%$ of patients, suggesting that even if T-DM1-based pre-operative treatment is administered, anthracyclines can be avoided in $40 \%$ of the cases. However, further confirmation of long-term prognosis with anthracycline-free adjuvant therapy is needed.

We observed a remarkably high pCR rate in group B and hypothesize that the HER2+ cancer cells/clones that persisted [19] after the initial 4 cycles of TCbHP treatment may have responded to the subsequent 4 cycles of T-DM1+P. Interestingly, this sequence of $\mathrm{TCbHP} \rightarrow \mathrm{T}-\mathrm{DM} 1+\mathrm{P}$ increased the $\mathrm{pCR}$ rate compared with the standard regimen of TCbHP, particularly for luminal HER2+ disease.

Although direct comparisons cannot be made with other studies due to differences in study design, region, and treatment regimens, the TRYPHAENA study reported a pCR rate (ypT0-isypN0) of $50.7 \%$ with the $\mathrm{FEC}+\mathrm{H}+\mathrm{P} \times 3 \rightarrow \mathrm{T}+\mathrm{H}+\mathrm{P} \times 3$ regimen, $45.3 \%$ with the $\mathrm{FEC} \times 3 \rightarrow \mathrm{T}+\mathrm{H}+\mathrm{P} \times 3$ regimen, and $51.9 \%$ with the $\mathrm{TCH}+\mathrm{P} \times 6$ regimen, the only comparable arm, compared with $57 \%$ in group A of this study [8]. The pCR rates in the ADAPT study were $41.0 \%$ for the T-DM1 $\times 4$ regimen and $41.5 \%$ for the T-DM1+ET $\times 4$ regimen in patients with early HER2+ HR + breast cancer [20]. However, in ADAPT, patients received 4 treatment cycles unlike 6 in our study. In the KRISTINE study, pCR was achieved in $44.4 \%$ (HR+, $38.1 \%$; HR-, $54.8 \%$ ) of patients in the T-DM1+P $\times 6$ group compared with $57.4 \%$ in group $\mathrm{C}$ in this study, and in $55.7 \%$ $(46.4 \%, 71.1 \%)$ of patients in the $\mathrm{TCbH}+\mathrm{P} \times 6$ group, which was similar to group A of this study (57\%) [10]. However, it should be noted that in our study, patients with ER+ breast cancer were treated with concurrent HT along with $\mathrm{T}-\mathrm{DM} 1+\mathrm{P}$, which could have additionally impacted the $\mathrm{pCR}$ rates. In an analysis of the I-SPY2 trial in invasive breast cancer in HER2+ subsets, neoadjuvant T-DM1+P $\times 4$ followed by doxorubicin + cyclophosphamide $\times 4$ resulted in a pCR rate of $52 \%(\mathrm{HR}+, 46 \%$; $\mathrm{HR}-, 64 \%)$ versus $22 \%$ (HR+, 17\%; HR-, 33\%) with taxane (paclitaxel) + trastuzumab only. The results were similar to the response-guided group C and their ER status for this study [9]. With regard to the DFS and OS outcomes, less than 3 events of recurrence and no events of death were observed at the 3-year follow-up in group C treated with the T-DM1-based regimen. This good outcome is also supported by the results of the KRISTINE trial (T-DM1+P versus TCH $+\mathrm{P})[21]$ and can be expected to spur the development of T-DM1-based pre-operative therapy. Similar to the current study, T-DM1based regimens demonstrated favorable results in patients with HER2+ metastatic breast cancer in previous studies, including TDM4450g [22] and EMILIA [23]; the efficacy of T-DM1 was more evident in patients with high IHC (3+) [21] or HER2-mRNA [21, 23] expression.

The efficacy of T-DM1 is also supported by the KATHERINE study, where the interim analysis shows that the invasive DFS was significantly higher in the T-DM1 group than the trastuzumab alone group among patients with HER2+ early breast cancer with residual invasive disease after completion of neoadjuvant therapy [24].

In pre-menopausal women with ER+, ovarian suppression is often engaged using HT. In the ADAPT study, pCR rates were $41.5 \%$ and $15.1 \%$ in patients with HER2+ HR+ breast cancer receiving concomitant HT with 12-week T-DM1 and trastuzumab, respectively [20], suggesting improved efficacy of anti-HER2 therapy without any detrimental effects with concomitant HT. In the Neo-LaTH study [25], patients receiving anti-HER2 therapy (with/without HT) before chemotherapy for longer duration (18 versus 6 weeks) tended to show more tumor shrinkage. In our study as well, pCR was the highest (69\%) in patients with ER+ in group B that received T-DM1 with concurrent HT, which was preceded by 4 cycles of TCbHP. Thus, de-escalation strategies using an antibody-drug conjugate regimen along with concomitant HT to enhance treatment efficacy warrant further investigation.

This approach of imaging-based and biopsy-incorporated treatment personalization may be useful for both maintaining $\mathrm{pCR}$ rates and minimizing toxicity. Further evaluation is required to support the initiation of the T-DM1+P with or without HT regimen, which may be useful in a selected group of HER2+ patients based on lack of feasibility.

As anticipated, patients with strong HER2 expression (IHC3+) had higher pCR rates than those with mild HER2 expression (IHC2+ and DISH+) in group $\mathrm{C}$ and subgroup $\mathrm{C} 1$, suggesting that the effect of T-DM1 might depend on membrane HER2 expression levels.

Breast conservation was achieved in approximately half (51-54\%) of the patients, except in subgroup C2 (38\%). 
Also, among those who underwent BCS instead of planned mastectomy, breast conservation success rate was higher in all patients (32-39\%), except subgroup C2 (14\%). These findings may be useful for considering surgical approaches for each individual. As anticipated, compared to the T-DM1 backbone only arm (group C), the incidence of AEs was highest in the taxane backbone arm (group A) followed by the taxane + T-DM1 backbone arm (group B). The T-DM1 backbone arm also reported better quality of life in the Swedish PREDIX HER2 trial (T-DM1 versus DTP) [26] and the KRISTINE trial (T-DM1+P versus TCH+P) [21]. Of special mention are drug-related alopecia and febrile neutropenia, which were less frequent in subgroup $\mathrm{C} 1(5.0 \%, 0 \%)$ versus group A $(94.1 \%, 21.6 \%)$, group B $(86.5 \%, 15.4 \%)$, or subgroup C2 $(81 \%, 33.3 \%)$. This suggests that tailoring the T-DM1 backbone into the neoadjuvant therapy regimens is beneficial from a safety perspective and the treatment of HER2+ breast cancer can be modified with a more personalized approach. Study limitations include the fact that the pharmacoeconomic efficiency of the regimens and impact of the $\mathrm{pCR}$ rate of the T-DM1 regimen on long-term survival and quality of life were not evaluated.

\section{Conclusion}

Overall, the pCR rate was not statistically different among the treatment groups. However, compared with the standard $\mathrm{TCbHP}$ and response-guided $\mathrm{T}-\mathrm{DM} 1+\mathrm{P}$ regimens, the $\mathrm{pCR}$ rate with the TCbHP $\rightarrow$ T-DM1+P regimen was numerically higher in the neoadjuvant setting. Exploratory analyses showed that the pCR rate was significantly higher with the TCbHP $\rightarrow$ T-DM1+P regimen than with the standard TCbHP and T-DM1+P regimens in patients with $\mathrm{ER}+$, but was comparable in ER- patients. ER status might be an indicator to personalize dual HER2-targeted therapy. T-DM1+P was generally safe and well tolerated, with the fewest AEs throughout the study. No significant differences in safety were observed between patients who received TCbHP alone and TCbHP $\rightarrow \mathrm{T}-\mathrm{DM} 1+\mathrm{P}$. Given the patient numbers, the personalization of treatment showed that the efficacy among $\mathrm{T}-\mathrm{DM} 1+\mathrm{P}$ regimen responders was better than that of the TCbHP regimen alone and could thus serve as a reasonable approach to minimize toxicity while maintaining efficacy.

Acknowledgements The manuscript data were presented in a poster at the European Society for Medical Oncology (ESMO) Annual Congress, September 8-12, 2017, Madrid, Spain. Editorial support, in the form of medical writing, assembling tables and creating high-resolution images based on authors' detailed directions, collating author comments, copyediting, fact checking, and referencing, was provided by Annirudha Chillar, MD, PhD, and Shama Buch, PhD, of Cactus Communications, and funded by Japan Breast Cancer Research Group. We would like to thank ICON JAPAN K.K. for managing the data collection; Satoshi Morita for the data analyses; EPS Corporation for site monitoring; and Kyoto University, ICON JAPAN K.K., and the coordinating investigators (Masakazu Toi and Norikazu Masuda) for the overall coordination of the trial. The authors thank all the sites that participated in the study (Online Resource 2) and Akira Shimizu (Department of Experimental Therapeutics Institute for Advancement of Clinical and Translational Science, Kyoto University Hospital), Yasuhiro Fujiwara, Takashi Inamoto, Akira Yoshioka, and Hajime Abe for their guidance in managing this physician-driven registration study.

Funding This work was supported by the Japan Breast Cancer Research Group (JBCRG). Funding was also provided by Chugai Pharmaceutical Co., Ltd.

Data availability Data archiving is not mandated but data will be made available on reasonable request.

\section{Compliance with ethical standards}

Conflict of interest NM has received honoraria from Chugai, AstraZeneca, Pfizer, Eli Lilly, Eisai, and Takeda; funding from Chugai, AstraZeneca, Kyowa Hakko Kirin, MSD, Novartis, Pfizer, Eli Lilly, Eisai, and Daiichi Sankyo; and is a board member of JBCRG. S. Ohtani has received honoraria from Chugai, Eisai, AstraZeneca, and Pfizer. T. Takano has received honoraria from Daiichi Sankyo, Kyowa Hakko Kirin, and Eisai; and research funding from Chugai, Taiho, Novartis, Takeda, Ono, MSD, Merck Serono, Daiichi Sankyo, Kyowa Hakko Kirin, and Eisai. K. Inoue has received funding from Parexel, Puma Biotechnology, GlaxoSmithKline, Pfizer, Novartis, Chugai, Bayer, Daiichi Sankyo, MSD, and Eli Lilly. HB has received honoraria from Chugai, AstraZeneca, Eisai, Taiho, Novartis, Pfizer, Kyowa Hakko Kirin; and research funding from Taiho and Eisai. YI has received funding from Daiichi Sankyo, Chugai, Novartis, Parexel, EPS, MSD, AstraZeneca, Eli Lilly, Kyowa Hakko Kirin, Covance, Taiho, and A2 Healthcare. TY has received lecture fee from Chugai, Eisai, Novartis, Pfizer, and Taiho. KK has received honoraria from Taiho Pharmaceutical Co, Kyowa Hakko Kirin Co, Astellas Pharma Inc, Eisai Co, and Novartis Pharma K.K. T. Takasuka is an employee and owns stocks of Chugai Pharmaceutical. SM received consulting fees or honoraria from JBCRG and Chugai. S Ohno received consulting fees or honoraria from Chugai, AstraZeneca, Taiho, Pfizer, Novartis, Kyowa Hakko Kirin, and Eisai; and funding from Chugai, Taiho, Daiichi Sankyo, and Eisai. MT received honoraria for lecture from Chugai and Takeda and funding from Chugai. ES, RN, K Ishida, HY, HK, TS, and TRK do not have any conflicts of interest to declare.

Ethical approval The experiments in this study comply with the current laws of the country in which they were performed.

Informed consent Informed consent was obtained from all individual participants included in the study.

Research involving human participants and/or animals All procedures performed in studies involving human participants were in accordance with the ethical standards of the institutional and/or national research committee and with the 1964 Helsinki declaration and its later amendments or comparable ethical standards.

Open Access This article is licensed under a Creative Commons Attribution 4.0 International License, which permits use, sharing, adaptation, distribution and reproduction in any medium or format, as long as you give appropriate credit to the original author(s) and the source, provide a link to the Creative Commons licence, and indicate if changes were made. The images or other third party material in this article are 
included in the article's Creative Commons licence, unless indicated otherwise in a credit line to the material. If material is not included in the article's Creative Commons licence and your intended use is not permitted by statutory regulation or exceeds the permitted use, you will need to obtain permission directly from the copyright holder. To view a copy of this licence, visit http://creativecommons.org/licenses/by/4.0/.

\section{References}

1. NCCN Guidelines: Breast Cancer. https://www.nccn.org/store/ login/login.aspx?ReturnURL=https://www.nccn.org/profession als/physician_gls/pdf/breast.pdf. Accessed 23 February 2019

2. Japan Breast Cancer Society Clinical Practice Guideline for treatment of breast cancer, 2018 edition [available in Japanese]. https ://jbcs.gr.jp/guidline/2018/. Accessed 23 February 2019

3. Baselga J, Bradbury I, Eidtmann H et al (2012) Lapatinib with trastuzumab for HER2-positive early breast cancer (NeoALTTO): a randomised, open-label, multicentre, phase 3 trial. Lancet 379(9816):633-640. https://doi.org/10.1016/S0140 -6736(11)61847-3

4. Robidoux A, Tang G, Rastogi P et al (2013) Lapatinib as a component of neoadjuvant therapy for HER2-positive operable breast cancer (NSABP protocol B-41): an open-label, randomised phase 3 trial. Lancet Oncol 14(12):1183-1192. https://doi.org/10.1016/ S1470-2045(13)70411-X

5. Kawaguchi K, Suzuki E, Kataoka TR, Hirata M, Ohno S, Bando $\mathrm{H}$, Ishiguro H, Inoue K, Yamamoto N, Kuroi K, Morita S, Masuda N, Toi M (2016) Analysis of tumor infiltrating lymphocytes in HER2-positive primary breast cancer treated with neoadjuvant lapatinib and trastuzumab: the NeoLath study (JBCRG-16). J Clin Oncol 15:599. https://doi.org/10.1200/JCO.2016.34.15_supp1.599

6. Iwata H, Yamamoto N, Masuda N, Bando H, Kuroi K, Ohno S, Kasai H, Morita S, Sakurai T, Toi M; JBCRG-16 (Neo-LaTH) Study Group (2015) P203 Dual HER2 blockage with lapatinib and trastuzumab for Japanese patients with HER2+ breast cancer. Breast 24:S95. https://doi.org/10.1016/S0960-9776(15)70237-7

7. Gianni L, Pienkowski T, Im YH et al (2012) Efficacy and safety of neoadjuvant pertuzumab and trastuzumab in women with locally advanced, inflammatory, or early HER2-positive breast cancer (NeoSphere): a randomised multicentre, open-label, phase 2 trial. Lancet Oncol 13(1):25-32. https://doi.org/10.1016/S1470 -2045(11)70336-9

8. Schneeweiss A, Chia S, Hickish T, Harvey V, Eniu A, Hegg R, Tausch C, Seo JH, Tsai Y-F, Ratnayake J, McNally V, Ross G, Cortés J (2013) Pertuzumab plus trastuzumab in combination with standard neoadjuvant anthracycline-containing and anthracyclinefree chemotherapy regimens in patients with HER2-positive early breast cancer: a randomized phase II cardiac safety study (TRYPHAENA). Ann Oncol 24(9):2278-2284. https://doi.org/10.1093/ annonc/mdt 182

9. DeMichele AM, Moulder S, Buxton M et al (2016) CT042: Efficacy of T-DM1+pertuzumab over standard therapy for HER2+ breast cancer: results from the neoadjuvant I-SPY2 trial. Cancer Res. https://doi.org/10.1158/1538-7445.AM2016-CT042

10. Hurvitz SA, Martin M, Symmans WF et al (2018) Neoadjuvant trastuzumab, pertuzumab, and chemotherapy versus trastuzumab emtansine plus pertuzumab in patients with HER2-positive breast cancer (KRISTINE): a randomised, open-label, multicentre, phase 3 trial. Lancet Oncol 19(1):115-126. https://doi.org/10.1016/ S1470-2045(17)30716-7

11. Johnston S, Pippen J Jr, Pivot X, Lichinitser M, Sadeghi S, Dieras V, Gomez HL, Romieu G, Manikhas A, Kennedy MJ, Press MF, Maltzman J, Florance A, O'Rourke L, Oliva C, Stein S, Pegram
M (2009) Lapatinib combined with letrozole versus letrozole and placebo as first-line therapy for postmenopausal hormone receptor-positive metastatic breast cancer. J Clin Oncol 27(33):55385546. https://doi.org/10.1200/JCO.2009.23.3734

12. Rimawi MF, Mayer IA, Forero A, Nanda R, Goetz MP, Rodriguez AA, Pavlick AC, Wang T, Hilsenbeck SG, Gutierrez C, Schiff R, Osborne CK, Chang JC (2013) Multicenter phase II study of neoadjuvant lapatinib and trastuzumab with hormonal therapy and without chemotherapy in patients with human epidermal growth factor receptor 2-overexpressing breast cancer: TBCRC 006. J Clin Oncol 31(14):1726-1731. https://doi.org/10.1200/ JCO.2012.44.8027

13. Kaufman B, Mackey JR, Clemens MR, Bapsy PP, Vaid A, Wardley A, Tjulandin S, Jahn M, Lehle M, Feyereislova A, Révil C, Jones A (2009) Trastuzumab plus anastrozole versus anastrozole alone for the treatment of postmenopausal women with human epidermal growth factor receptor 2-positive, hormone receptorpositive metastatic breast cancer: results from the randomized phase III TAnDEM study. J Clin Oncol 27(33):5529-5537. https ://doi.org/10.1200/JCO.2008.20.6847

14. Rimawi M, Ferrero JM, de la Haba-Rodriguez J, Poole C, De Placido S, Osborne CK, Hegg R, Easton V, Wohlfarth C, Arpino G; PERTAIN Study Group (2018) First-line trastuzumab plus an aromatase inhibitor, with or without pertuzumab, in human epidermal growth factor receptor 2-positive and hormone receptor-positive metastatic or locally advanced breast cancer (PERTAIN): a randomized, open-label phase II trial. J Clin Oncol 36(28):2826-2835. https://doi.org/10.1200/JCO.2017.76.7863

15. Fleeman N, Bagust A, Boland A, Dickson R, Dundar Y, Moonan M, Oyee J, Blundell M, Davis H, Armstrong A, Thorp N (2011) Lapatinib and trastuzumab in combination with an aromatase inhibitor for the first-line treatment of metastatic hormone receptor-positive breast cancer which over-expresses human epidermal growth factor 2 (HER2): a systematic review and economic analysis. Health Technol Assess 15(42):1-93. https://doi.org/10.3310/ hta 15420

16. von Minckwitz G, Untch M, Blohmer JU et al (2012) Definition and impact of pathologic complete response on prognosis after neoadjuvant chemotherapy in various intrinsic breast cancer subtypes. J Clin Oncol 30(15):1796-1804. https://doi.org/10.1200/ JCO.2011.38.8595

17. Wolff AC, Hammond ME, Hicks DG et al (2013) Recommendations for human epidermal growth factor receptor 2 testing in breast cancer: American Society of Clinical Oncology/ College of American Pathologists clinical practice guideline update. J Clin Oncol 31(31):3997-4013. https://doi.org/10.1200/ JCO.2013.50.9984

18. Kuroi K, Toi M, Ohno S, Nakamura S, Iwata H, Masuda N, Sato N, Tsuda H, Kurosumi M, Akiyama F (2015) Comparison of different definitions of pathologic complete response in operable breast cancer: a pooled analysis of three prospective neoadjuvant studies of JBCRG. Breast Cancer 22(6):586-595. https://doi. org/10.1007/s12282-014-0524-4

19. Kim C, Gao R, Sei E, Brandt R, Hartman J, Hatschek T, Crosetto N, Foukakis T, Navin NE (2018) Chemoresistance evolution in triple-negative breast cancer delineated by single-cell sequencing. Cell 173(4):879-893. https://doi.org/10.1016/j.cell.2018.03.041

20. Harbeck N, Gluz O, Christgen M et al (2017) De-escalation strategies in human epidermal growth factor receptor 2 (HER2)-positive early breast cancer (BC): final analysis of the West German Study Group Adjuvant Dynamic Marker-Adjusted Personalized Therapy Trial Optimizing Risk Assessment and Therapy Response Prediction in Early BC HER2- and hormone receptor-positive phase II randomized trial-efficacy, safety, and predictive markers for 12 weeks of neoadjuvant trastuzumab emtansine with or without 
endocrine therapy (ET) versus trastuzumab plus ET. J Clin Oncol 35(26):3046-3054. https://doi.org/10.1200/JCO.2016.71.9815

21. Hurvitz SA, Martin M, Jung KH et al (2019) Neoadjuvant trastuzumab emtansine and pertuzumab in human epidermal growth factor receptor 2-positive breast cancer: three-year outcomes from the phase III KRISTINE study. J Clin Oncol 37(25):2206-2216. https://doi.org/10.1200/JCO.19.00882

22. Hurvitz SA, Dirix L, Kocsis J, Bianchi GV, Lu J, Vinholes J, Guardino E, Song C, Tong B, Ng V, Chu YW, Perez EA (2013) Phase II randomized study of trastuzumab emtansine versus trastuzumab plus docetaxel in patients with human epidermal growth factor receptor 2-positive metastatic breast cancer. J Clin Oncol 31(9):1157-1163. https://doi.org/10.1200/JCO.2012.44.9694

23. Baselga J, Lewis Phillips GD, Verma S, Ro J, Huober J, Guardino AE, Samant MK, Olsen S, de Haas SL, Pegram MD (2016) Relationship between tumor biomarkers and efficacy in EMILIA, a Phase III study of trastuzumab emtansine in HER2-positive metastatic breast cancer. Clin Cancer Res 22(15):3755-3763. https:// doi.org/10.1158/1078-0432.CCR-15-2499

24. von Minckwitz G, Huang CS, Mano MS et al (2019) Trastuzumab emtansine for residual invasive HER2-positive breast cancer. $\mathrm{N}$ Engl J Med 380(7):617-628. https://doi.org/10.1056/NEJMo a1814017

\section{Affiliations}

\section{Norikazu Masuda' ${ }^{1}$ (D) Shoichiro Ohtani ${ }^{2} \cdot$ Toshimi Takano $^{3} \cdot$ Kenichi Inoue $^{4} \cdot$ Eiji Suzuki $^{5} \cdot$ Rikiya Nakamura $^{6}$. Hiroko Bando ${ }^{7}$. Yoshinori Ito ${ }^{8} \cdot$ Kazushige Ishida $^{9} \cdot$ Takashi Yamanaka $^{10} \cdot$ Katsumasa Kuroi $^{11}$. Hiroyuki Yasojima ${ }^{1}$. Hiroi Kasai ${ }^{12} \cdot$ Tsuyoshi Takasuka $^{13} \cdot$ Takaki Sakurai $^{14} \cdot$ Tatsuki R. Kataoka $^{14}$ - Satoshi Morita ${ }^{15} \cdot$ Shinji Ohno $^{16}$. Masakazu Toi ${ }^{17}$}

1 Department of Surgery, Breast Oncology, National Hospital Organization Osaka National Hospital, Osaka, Japan

2 Department of Breast Surgery, Hiroshima City Hiroshima Citizens Hospital, Hiroshima, Japan

3 Department of Medical Oncology, Toranomon Hospital, Tokyo, Japan

4 Division of Breast Oncology, Saitama Cancer Center, Saitama, Japan

5 Department of Breast Surgery, Kyoto University Hospital, Kyoto, Japan

6 Division of Breast Surgery, Chiba Cancer Center, Chiba, Japan

7 Breast and Endocrine Surgery, Faculty of Medicine, University of Tsukuba, Tsukuba, Japan

8 Breast Medical Oncology Department, Cancer Institute Hospital of Japanese Foundation for Cancer Research, Tokyo, Japan

9 Department of Surgery, Iwate Medical University, Morioka, Japan
25. Masuda N, Toi M, Yamamoto N, Iwata H, Kuroi K, Bando H, Ohtani S, Takano T, Inoue K, Yanagita Y, Kasai H, Morita S, Sakurai T, Ohno S (2018) Efficacy and safety of trastuzumab, lapatinib, and paclitaxel neoadjuvant treatment with or without prolonged exposure to anti-HER2 therapy, and with or without hormone therapy for HER2-positive primary breast cancer: a randomised, five-arm, multicentre, open-label phase II trial. Breast Cancer 25(4):407-415. https://doi.org/10.1007/s1228 2-018-0839-7

26. Brandberg Y, Andersson A, Bjohle J, Bosch A, Carlsson L, Dreifaldt AC (2019) Health-related quality of life in the Swedish PREDIX HER2 trial, evaluating docetaxel, trastuzumab, pertuzumab versus trastuzumab emtansine as neoadjuvant treatment of HER2-positive breast cancer. J Clin Oncol 37:583. https://doi. org/10.1200/JCO.2019.37.15_suppl.583

Publisher's Note Springer Nature remains neutral with regard to jurisdictional claims in published maps and institutional affiliations.
10 Department of Breast and Endocrine Surgery, Kanagawa Cancer Center, Yokohama, Japan

11 Department of Breast Surgery, Tokyo Metropolitan Health and Hospitals Corporation Ebara Hospital, Tokyo, Japan

12 Institute for Advancement of Clinical and Translational Science, Kyoto University Hospital, Kyoto, Japan

13 Oncology Lifecycle Management Department, Chugai Pharmaceutical Co., Ltd., Tokyo, Japan

14 Department of Diagnostic Pathology, Kyoto University Hospital, Kyoto, Japan

15 Department of Biomedical Statistics and Bioinformatics, Kyoto University Graduate School of Medicine, Kyoto, Japan

16 Breast Oncology Center, Cancer Institute Hospital of Japanese Foundation for Cancer Research, Tokyo, Japan

17 Breast Cancer Unit, Kyoto University Hospital, Kyoto University, 54 Shogoin-Kawaharacho, Sakyo-ku, Kyoto 606-8507, Japan 